\title{
Review on Protocol based Approaches to Extend Lifetime of Wireless Sensor Networks
}

\author{
Sharad Saxena \\ Assistant Professor, \\ Department of Computer \\ Application, Bharat Institute of \\ Technology, \\ Meerut, India.
}

\author{
Dr. Shailendra Mishra \\ Professor and Head, CSE \\ Department, Kumaun \\ Engineering College, \\ Dwarahat, District \\ Almorah, India.
}

\author{
Dr. A. K. Sharma \\ Professor and Head, \\ YMCA Engineering \\ College, \\ Faridabad, India.
}

\author{
Dr. D. S. Chauhan \\ Vice Chancellor, \\ Uttarakhand Technical \\ University, \\ Dehradun, India.
}

\begin{abstract}
Researchers in wireless Micro-sensor networks (M-WSN) have proposed various protocols for energy conservations. Each protocol is aimed to optimize power utilization in wireless sensor networks. The different protocol approaches optimize power consumption either considering their application areas or network topologies. This paper studies various energy-saving protocols for micro sensor networks and presents their brief classifications. An M-WSN is a group of hundreds or thousands of small energy-limited sensors that are densely deployed in a large geographical region. These micro sensors in sensor network are autonomous devices responsible for forwarding locally collected data to a central node called sink node by following multi-hop wireless paths. The sink node performs data fusion to form a single meaningful result. The micro sensors work by utilizing limited battery-power, the considerable part is to use this power for a maximum time. Although recent developments in electronics has enabled the development of low-cost \& low-power sensors networks, still there is a challenging job of energy conservation optimization within the wireless sensor networks (WSNs).
\end{abstract}

Keywords: sensor network, duty cycle, protocol, data aggregation, fusion.

\section{INTRODUCTION}

A collection of widely deployed sensors over a geographical region is known as micro sensor network. This network can be used to fulfill various objectives like monitoring activities, vibration sensing, humidity measurement etc. A micro sensor is very small in size, still it has three components: a sensing system for data collection, a power supply (battery) and a processing system to process the data. Since batteries are very small in size, it has limited storage of charge and can work for a short time frame. Recharging or replacing these batteries is very difficult as they are deployed in a hostile environment. Some application specific sensors must fulfill their requirements for a longer time, therefore the typical work is to enhance the sensor network life time by enhancing or optimizing power needs of the sensors. However solar cells can be used as external resource [15] in some specific applications. This paper considers mainly the micro sensor network model shown in Figure 1 and consisting of one sink node (base station) and a (large) number of micro sensors deployed over a large geographic area (sensing field). Data are transferred from sensor nodes to the sink through a multi-hop communication paradigm [16]. Out of three major components of sensor, the data processing part consumes less power as compared to data transmission from one end point to another end point [36]. Processing cost of thousand bits is approximately same as consumed in transmission of a single bit. The energy saving techniques thus considers two aspects; different network protocols designs and minimization of the frequency of energy expensive samples.

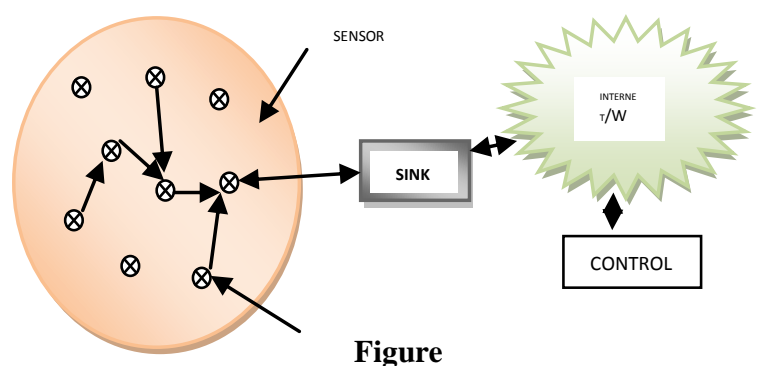

Figure 1: Sensor Network Architecture

Different other methods [12] like design of energy efficient communication protocols, switching on/off the radio when not in use are also explored. For example, design of energyefficient protocols to minimize the energy consumption during network activities; switching off node components that are not temporarily needed etc. This paper surveys the main protocol based techniques used for energy conservation in wireless sensor networks.

\section{DUTY CYCLING PROTOCOLS FOR ENERGY CONSERVATION}

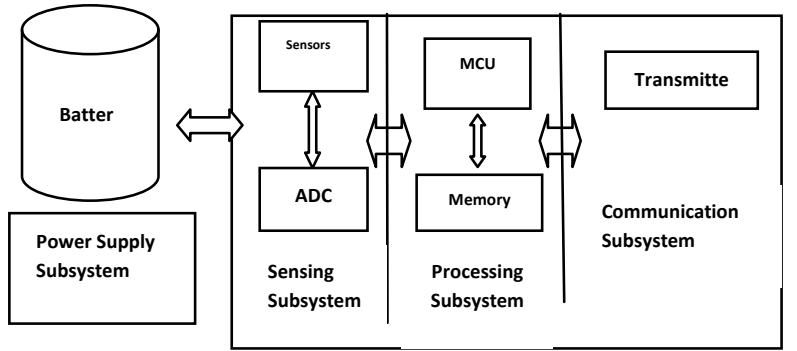

Figure 2: Architecture of typical wireless sensor node Figure 2, above shows the architecture of a typical wireless micro sensor. The four essential components of the 
architecture are: (a) a sensing subsystem having sensor along with analog-to-digital (ADC) converters for data acquisition; (b) a processing subsystem including a micro-controller (MCU) and memory for local data processing; (c) a transmitter subsystem for wireless data communication; and (d) a power supply unit (battery). Duty cycling concept is related to radio transceiver state of a sensor, the idea is to keep it off when it is not in use and on when a sensor need to transmit a data. Hence the state changes from sleep to active and vice versa. This can be achieved by selecting a subset of nodes among the entire sensors to remain active and rest of them as sleep. Selection of few nodes for activation is known as topology control and duty cycling operated on active nodes are known as power management. Figure 3 shows various powers management protocols that can be implemented either as independent sleep/wakeup protocols or can be integrated with the MAC protocol. Integration with MAC can optimize medium access functions based on the specific sleep/wakeup pattern used for power management while, independent sleep/wakeup protocols permit a greater flexibility as they can be tailored to the application needs.

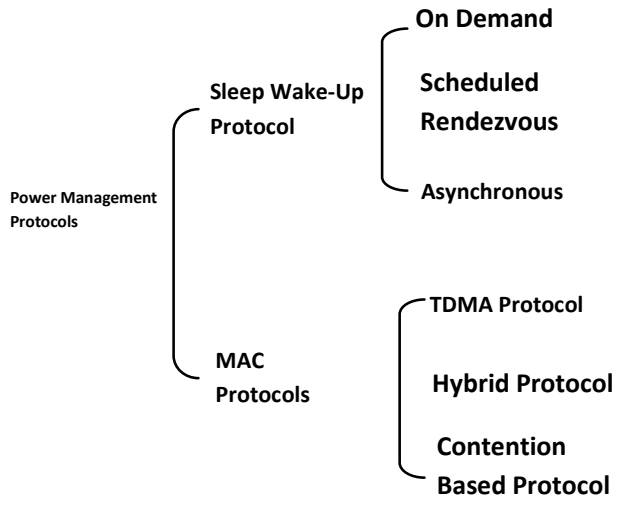

Figure 3: Protocol based Energy saving approaches

The various approaches are explained below briefly.

\subsection{Sleep/Wakeup protocols}

The sleep/wakeup protocols are related to radio subsystem of a sensor without considering the topology or connectivity issues. The sleep/wakeup pattern is possible in three ways as on demand, rendezvous way or in asynchronous manner. Hence they are classified in three possible categories [33]: OnDemand, Scheduled Rendezvous and Asynchronous Scheme. On-demand method depicts that a node should be active only when another node want to interact with it, but the problem here is how the sender node inform the receiver that it want to communicate with it. The possible proposed solution for it is the use of more than one radio with different energy consumption ratio. The high power radio is used for data transmission while, the low power radio for signaling purpose. In rendezvous scheme each node wake up, and remain active, at the same time as its neighbors according to a wakeup schedule. Then, they go to sleep for the next rendezvous time. Finally, an asynchronous sleep/wakeup protocol uses an asynchronous protocol to wake up a node, when it wants to communicate with its neighbors.

\subsubsection{On-demand schemes}

On-demand scheme focuses on awakening of a node when it has a packet to receive from a neighbor. This is applicable where the sensor networks have a very low duty cycle e.g. fire detection system etc. Here the sensors spent their most of the time in monitoring the environment. As soon as an event is detected, nodes become active to awaken state. The sensors here are equipped with two different channels, one for normal data communication and second for awaking sensor nodes. Implementation of second radio incurred an additional cost, which is however very small as compared to the cost of sensor node. The critical aspect is the mismatch between the coverage of two radios. Sparse Topology and Energy Management (STEM) [7] uses the approach of two different radios for wakeup signal and data packet transmissions, respectively. Here the wakeup radio is not a low power radio; it also needs an asynchronous duty cycle scheme. Each node periodically turns on its wakeup radio for some duration. When a source node has to communicate with a neighboring node, it sends a stream of periodic beacons (STEM-B) on the wakeup channel. Target node receives a beacon and sends back a wakeup acknowledgement, and turns on its data radio. In case of a collision no wakeups acknowledgement is sent. The wakeup beacon is retransmitted randomly unless a wakeup acknowledgement is received from target node. In [8] author proposes a variant of STEM-B as STEM-T, where a wakeup tone is used instead of a beacon. Both STEM-B and STEM-T can be used in combination with topology control protocols. The inter-beacon period in STEM is large enough to send and receive wakeup beacon. If $\mathrm{T}_{\text {wakeup }}$ and $\mathrm{T}_{\text {wack }}$ denote the time required to transmit a wakeup beacon and the related acknowledgement then, the receiver must listen on the wakeup radio for a time $T_{\text {active }}=T_{\text {wakeup }}+T_{\text {wack }}$ to ensure the correct reception of the beacon. Here, $\mathrm{T}_{\text {active }}$ depends on the bit rate of network node and in low bit-rate networks the time between successive active periods must be very large to allow a low duty cycle on the wakeup channel. In multi-hop networks it results in large wakeup latency. In [39] the author has proposed a Pipelined Tone Wakeup (PTW) scheme to deal with energy saving and wakeup latency. PTW uses two different channels for transmitting wakeup signals and data packets, and a wakeup tone to awaken neighbor's nodes. Sender in PTW takes care of tone detection. This enables the duration of wakeup tone long enough to be detected by the receiver that turns on its radio periodically. The rationale behind this solution is that the sender only sends a wakeup tone when an event is detected, while receivers wake up periodically. In addition, the wakeup procedure is pipelined with the packet transmission so as to reduce the wakeup latency and hence, the overall message latency. The idea is illustrated in Figure 5 with reference to the string topology network depicted in Figure. 4. This tone awakens all A's neighbors. At time $\mathbf{t}_{\mathbf{1}} \mathrm{A}$ sends a notification packet to $\mathrm{B}$ on the data channel to inform that the next data packet will be destined to B.

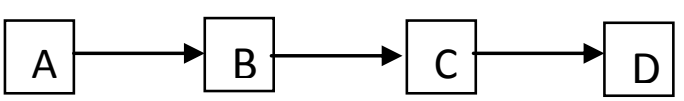

Figure 4: String Topology 


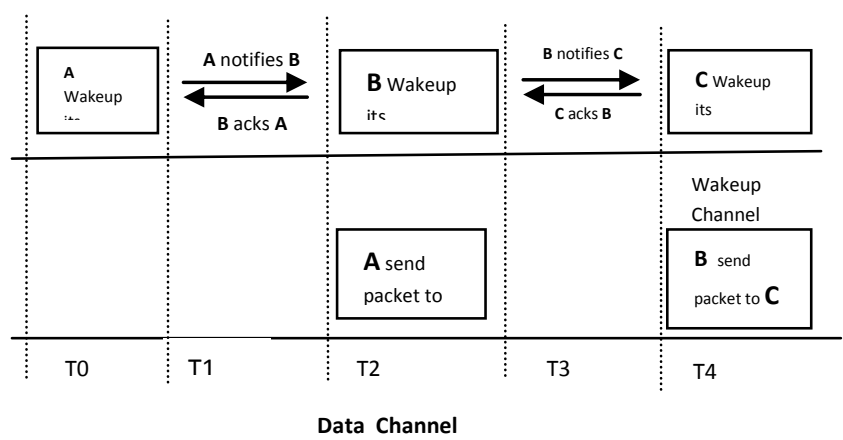

Figure 5: PTW pipelined wakeup procedure

Upon receiving the notification message $B$ learn that the following message is not intended for them. Therefore, they turn off their data radio. Instead, $B$ realizes to be the destination of next data message and replies with a wakeup acknowledgment on the data channel. Then A starts transmitting the data packet on the data channel. At the same time, B starts sending a tone on the wakeup channel to awake all its neighbors. As shown in Figure. 4 the packet transmission from A to B on the data channel and the B's tone transmission on the wakeup channel are done concurrently. A different approach is using a low power radio for the wakeup channel. The low-power radio is continuously in stand-by, and whenever receives a signal it wakes up the data radio $[6,18]$. The wakeup latency is thus minimized. The main drawback of this approach is that the transmission range of the wakeup radio is significantly smaller than that of the data radio. This may limit the applicability of such a technique as a node may not be able to wake up a neighboring node even if it is within its data transmission range. A side effect of using a second radio for the wakeup channel is the additional power consumption, which may not be negligible even when using a low-power radio. To overcome problems associated with the extra-energy consumed by the wakeup radio, a RadioTriggered Power Management scheme is investigated in [20]. The basic idea is to use the energy contained in wakeup messages (e.g. STEMB beacon) or signals (e.g., STEM-T and PTW tones) to trigger the activation of the sensor node. This approach is similar to the one used in active Radio Frequency Identification (RFID) systems [22]. In radio triggered power management method a special hardware component, a radiotriggered circuit, is used to capture the energy contained in the wakeup signal, and uses such energy to trigger an interrupt for waking up the node. The radio-triggered approach is significantly different from using a stand-by radio to listen to possible wakeup messages from neighboring nodes. The standby radio consumes energy from the node when listening, while the radio-triggered circuit is powered by the wakeup message. The main drawback of the radio-triggered approach is the limitation on the maximum distance from which the wakeup message can be sent. With the basic radio-triggered circuit proposed in [20], the maximum achievable distance is $3 \mathrm{~m}$. However this distance may be increased up to certain extent with additional cost.

\subsubsection{Scheduled rendezvous wakeup schemes}

All the neighboring nodes wake up at the same time in this scheme. Waking up of node is periodic in which the node checks for communications and then return to sleep until the next rendezvous time. The good feature of this protocol is that when a node is awakened, it is guaranteed that all its neighbors are awake as well. This requires a broadcast message to send to all neighbors [33]. A clock synchronization scenario is needed for this kind of scheduling. Different scheduled rendezvous protocols differ in the way network nodes sleep and wake up during their lifetime. The simplest way is using a Fully Synchronized Pattern [3]. In this case all nodes in the network wake up at the same time according to a periodic pattern. More precisely, all nodes wake up periodically every $\mathrm{T}_{\text {wakeup }}$, and remain active for a fixed time $\mathrm{T}_{\text {active }}$. Then, they return to sleep until the next wakeup instant. Due to its simplicity this scheme is used in several practical implementations including TinyDB [31] and TASK [24]. A fully synchronized wakeup scheme is also used in MAC protocols such as S-MAC [38] and T-MAC [34]. Even if simple, this scheme allows a low duty cycle provided that the active time $\left(\mathrm{T}_{\text {active }}\right)$ is significantly smaller than the wakeup period $\left(\mathrm{T}_{\text {wakeup }}\right)$. A further improvement can be achieved by allowing nodes to switch off their radio when no activity is detected for at least a timeout value [34]. In addition, due to the large size of the active and sleeping part, it does not require very precise time synchronization [31]. The main drawback is that all nodes become active at the same time after a long sleep period. Therefore, nodes try to transmit simultaneously, thus causing a large number of collisions. In addition, the scheme is not very flexible since the size of wakeup and active periods is fixed and does not adapt to variations in the traffic pattern and/or network topology. The internal network organization and the change in network topology, as the nodes join or leave, needs node balancing. Some of the sleep/wakeup schemes take advantage of it. However, under the assumption that nodes are static, the data-gathering tree is supposed to remain stable for a reasonable amount of time [21]. In the Staggered Wakeup Pattern (SWP) [3], shown in Figure 5, nodes located at different levels of the data-gathering tree wake up at different times. Obviously, the active parts of nodes belonging to adjacent levels must be partially overlapped to allow nodes to communicate with their children.

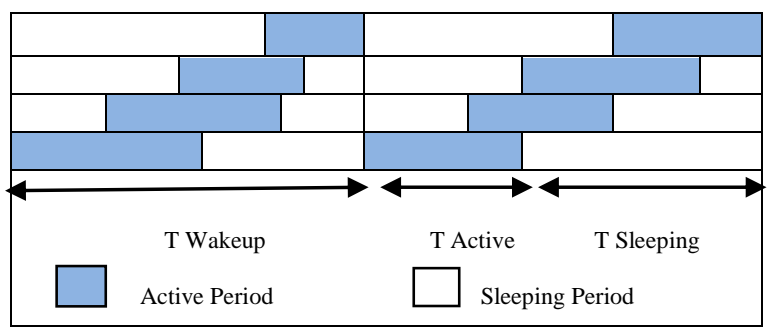

Figure 6: Staggered Sleep/Wakeup Pattern

The active parts of different levels are arranged in such a way that the portion of the active period a node uses to receive packets from its children is adjacent to the portion it uses to send packets to its parent (Figure. 6). This minimizes the energy dissipation for transitioning from sleep to active mode. The staggered wakeup pattern shown in Figure 6 is also called backward staggered pattern [3] as it optimizes packet latency in the backward direction i.e., from leaf nodes to the root. It is also possible to arrange nodes' active periods in such a way to optimize the forward packet latency (i.e., from the root to leaves). The resulting scheme, called forward staggered pattern [3] is however not very used in practice, because in real networks most of data flows from sensor nodes to the sink. A combination of the backward and forward staggered pattern is 
also possible. The (backward) staggered scheme was first proposed in the framework of TinyDB [31] and TAG [30]. A staggered wakeup pattern is also used in D-MAC [21]. The advantages of staggered approach are; since nodes at different levels of the data-gathering tree wake up at different times, at a given time only a (small) subset of nodes in the network will be active, hence reduction in the number of collisions. The active period of each node is thus significantly shortened with respect to the fully synchronized scheme and resulting in energy saving. This scheme is also suitable for data aggregation as parent nodes receive data from all their children before they forward such data to their own parent at the higher level. This allows parent nodes to filter data received from children, or to aggregate them. The drawback of this approach is that, since nodes located at the same level in the data gathering tree wake up at the same time, collisions can potentially still occur. In addition, this scheme has limited flexibility due to the fixed duration of the active $\left(\mathrm{T}_{\text {active }}\right)$ and wakeup ( $\mathrm{T}_{\text {wakeup }}$ ) periods. Ideally, the active period should be as low as possible, not only for energy saving but also for minimizing the latency experienced by packets to reach the root node (see Figure. 5). Still the changes in traffic pattern are possible because of change of topology by nodes movements. An adaptive and low latency staggered scheme is proposed in [13]. This adaptive scheme not only minimizes the energy consumption but also provides lower average packet latency with respect to a fixed staggered scheme. It also reduces collisions by allowing different lengths of the active period for nodes belonging to the same level, but associated with different parents [13]. Another approach derived from the on demand TDMA scheme, is taken in Flexible Power Scheduling (FPS) [37]. FPS takes a slotted approach, i.e. time is assumed to be divided in slots of duration Ts. Slots are arranged to form periodic cycles, where each cycle is made up of $\mathrm{m}$ slots and has a duration of $\mathrm{Tc}=\mathrm{m}$ Ts. Each node maintains a power schedule of what operations it performs during a cycle. A node must keep its own radio on only when it has to receive/transmit from/to other nodes. The two common problems with this scheme are: they are not flexible and require a strict synchronization among nodes and to achieve flexibility, FPS includes an on-demand reservation mechanism that allows nodes to reserve slots in advance. Twinkle that supports broadcast is an improved version of FPS, presented in [4]

\subsubsection{Asynchronous schemes}

Asynchronous schemes allow each node to wake up independently and there is a guaranteed overlapped active periods within a specified number of cycles. Quorum Based asynchronous wakeup protocol was introduced in [19] with reference to IEEE 802.11 ad hoc networks. The Quorum technique considers two aspects, first is wakeup prediction: where host need to know when it will turn its radio on. Secondly, neighbor discovery: as the hosts' transmission/reception activities are reduced under the power saving mode, a host may take longer time, or maybe even unable, to detect the arrival and departure of other hosts in its radio covered range. In [27] the author proposed the wakeup schedule using asynchronous wakeup mechanisms [10]. After analysis they designed an Asynchronous Wakeup Protocol (AWP), which can detect neighboring nodes in a finite time. It can also handle packet collisions and variations in the network topology. AWP utilizes a wakeup function to generate a wake schedule. The two nodes adjacent to each other must overlap their wakeup schedule, though there can be a difference in their clocks. Figure 7, below shows the idea, by means of an example of asynchronous wakeup schedule for a set of 7 neighboring nodes. This example is based on a symmetric (7, 3 , 1)-design of the wakeup schedule function. Symmetric means that all nodes have the same duty cycle, while $(7,3$, and 1)-design indicates that: (i).each schedule repeats every seven slots; (ii).each schedule has three active slots out of seven (dark slots); and (iii).any two schedules overlap for at most one slot.

As shown in Figure 7 below, by following its own schedule, each node is guaranteed to communicate with any other neighboring node. The proposed scheme takes care of the fact that each node will be able to contact any of its neighbors in a finite time.

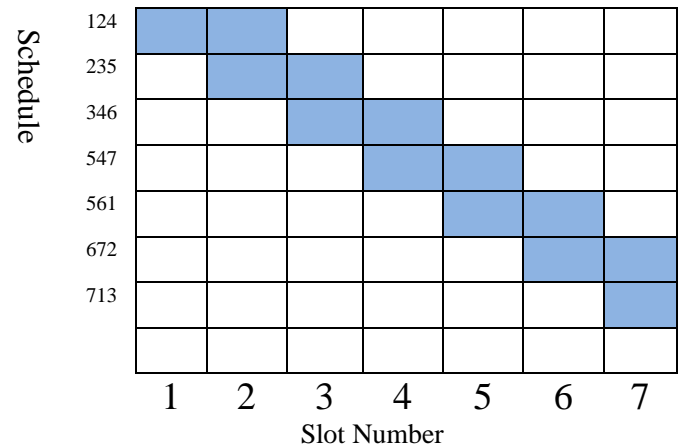

Figure 7: Asymmetric schedule based on $(7,3,1)$ function

In this scheme it never happens that all neighbors are simultaneously active. Therefore, it is not possible to broadcast a message to all neighbors [33]. Random Asynchronous Wakeup (RAW) [35] is another protocol that utilizes the fact that sensor networks are typically characterized by a high node density. This allows the existence of several paths between a source and a destination and, thus, a packet can be forwarded to any of such available paths. Actually, the RAW protocol consists of random wakeup scheme. The basic idea of the random wakeup scheme is that each node wakes up randomly once in every time interval of fixed duration $\mathrm{T}$ remains active for a predefined time Ta, and then sleeps again. Once awake, a node looks for active neighbors by running a neighbor discovery procedure. The random wakeup scheme is extremely simple and relies only on local decisions. This makes it well suited for networks with frequent topology changes. With RAW, it is not guaranteed that a node can find another active neighbor upon wakeup. Hence, RAW does not guarantee the packet forwarding within one time frame, while AWP does. An alternative approach to ensure that an asynchronous node finds its communication counterpart active when it wakes up, is forcing the receiver to listen periodically. The receiver wakes up periodically and listens for a short time to discover any potential asynchronous sender. If it does not detect any activity on the channel it returns to sleep, otherwise remains active to send/receive packets.

\subsection{MAC protocols}

The most common MAC protocols are classified as TDMA based, contention based, and hybrid protocols. Various MAC protocols are available in the literature [9, 26, 14 and 25]. 


\subsubsection{TDMA based MAC protocols}

TDMA allows time-slots exclusively assigned to each node for periodic transmission without facing any contention. TDMA is most desirable in WSNs as it saves energy by allowing a node to minimize idle listening. TDMA proposed in [28] for a sensor network determines the smallest conflict-free assignment of slots during which the packets generated at each node to reach their destination. They analyzed that when there is no loop in the network and has only one sink node, a minimum-delay scheduling can always be found. Their results showed a substantial reduction of energy and delay is possible. In [32] author proposes a simple minimum-delay schedule algorithm to find the slot lengths for all the links. Latency Energy Minimization Medium Access (LEMMA) [23] is an improvement to TDMA to reduce latency. LEMMA describes two states: transitory state that allocates time-slot during initialization phase and steady state, for data transmission. The latency is achieved by coupling a near-optimal cascading TDMA slot allocation with very low duty-cycle. Here the slot allocation algorithm tries to allot interference-free, time-slots with the highest number to nodes that are directly connected to sink node, and a similar approach is followed by each level of the tree, resulting a cascading assignment that minimize the latency required to transmit one packet from source to sink.

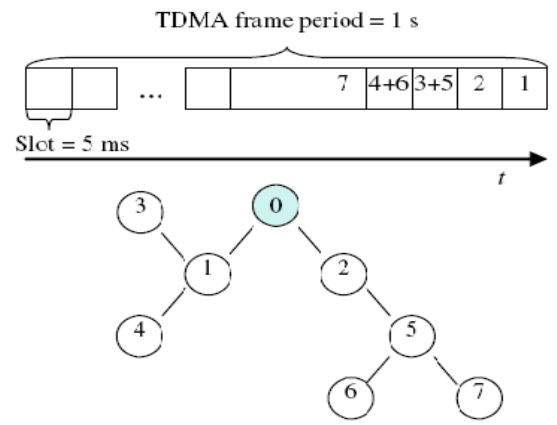

Figure 8: TDMA slot assignment for low-latency low dutycycle operation

Figure 8, shows an example, where the numbers inside the time-slots represent the node assignment for data transmission. In this example a TDMA frame of duration 1 second is divided in 200 time-slots of 5 milliseconds. To each node only one slot is assigned, which it is used to transmit data to its parent in the converge cast tree (with the sink at node 0), but LEMMA supports multi-slot assignments as well. The example considers that nodes 5, 6 and 7 do not interfere with nodes 3 and 4 . This allows the same slots to be assigned to 3 and 5 and to 4 and 6 . Each time-slot must be long enough to allow at least one DATA/ACK exchange (considering the maximum length of DATA packets). Carrier sense is used for medium access in each slot to assure interference free transmission. LEMMA is also able to support very low duty-cycles, since each node must only active listen in the beginning of as many slots as the number of children.

\subsubsection{Hybrid MAC protocol}

Hybrid MAC protocols behaves like both TDMA and CSMA, depending on the level of contention. The Probabilistic TDMA (PTDMA) [2] is a hybrid protocol where time is slotted, and nodes are distinguished in owners and non-owners. The protocol determines the accessibility of owners and non- owners depending on the number of senders. By doing so it adapts the MAC protocol to a TDMA or a CSMA scheme depending on the level of contention in the network. PTDMA does not take into account issues such as topology changes, synchronization errors, interference irregularities in to consideration, which are quite common in wireless sensor networks.

Z-MAC [17], another hybrid protocol uses neighbor discovery protocol and builds a list of two hop neighbors. Then a distributed slot assignment algorithm is applied to ensure that any two nodes in the two-hop neighborhood are not assigned to the same slot. Hence interference of node communications is avoided. Z-MAC does not use a global frame equal for all nodes since it is very difficult and expensive to adapt as sensor network topology changes. In Z-MAC each node maintains its own local time frame depending on the number of neighbors. It helps avoiding conflict with its contending neighbors. The time frame and local slot assignment of each node is then forwarded to its two-hop neighbors. Thus any node has slot and frame information about any two-hop neighbors. This is used for synchronization purpose. After this setup phase nodes can be in one of the following modes: Low Contention Level (LCL) and High Contention Level (HCL). A node is in the LCL unless it has received an Explicit Contention Notification $(\mathrm{ECN})$ within the last TECN period. ECNs are sent by nodes when they experience high contention. In HCL only the owners of the current slot and their one-hop neighbors are allowed to compete for accessing the channel. In LCL any node (both owners and non-owners) can compete to transmit in any slot. However, the owners have priority over non owners. This way Z-MAC can achieve high channel utilization even under low contention because a node can transmit as soon as the channel is available.

\subsubsection{Contention based MAC protocols}

The same communication channel is shared by number of nodes in MAC protocol [5]. Therefore the collision in the channel is unavoidable. Most common example of it are CSMA protocol [1] and ALOHA protocol. In CSMA, a node listens to the channel before transmitting. If it detects a busy channel, it delays access and retransmit later. In ALOHA, a node simply transmits a packet whenever it is generated or at the next available time slot. The collided packets are discarded and re-transmitted later.

The constraint associated with MAC protocols are: Scalability and Adaptability that accommodate changes in network size and topology, Mobility that allows sensor network to adapt mobility patterns, Collision Avoidance that ensures when a node can access a channel, Latency is the time required to send a packet from sender to receiver, Reliability for reliable delivery of data, Throughput refers to the maximum size of data, Channel Utilization related to the bandwidth of the channel.

Collaborative MAC (CC-MAC) CC-MAC protocol [29] utilizes the fact that sensor node located near each other generate the correlated data. CC-MAC filters data from highly correlated sensor nodes so that a node must handle minimum of messages, hence saving energy. CC-MAC consists of two components: the event MAC (E-MAC), to filter sensor node measurements to reduce traffic and network MAC (N-MAC), to forward the filtered measurement to the sensor network sink. E-MAC allows only sensor nodes separated by at least the correlation distance to generate measurements and other 
nodes can sleep to save energy and periodically awake to forward messages. For this purpose the sensor nodes must possess ranging information about their neighbors, which is its' main drawback. Furthermore the complexity of the CCMAC protocol may limit the application of the protocol.

PAMAS (Power aware multi-access with signaling) protocol [32] saves battery power by switching off nodes that are not transmitting or receiving data. It uses two transceivers: one for control message and other for data messages. Control channel exchanges use RTS, CTS messages, and a busy tone. The busy tone indicates that the data channel is busy. Message transfer in PAMAS starts by the source sending an RTS message to the destination on the control channel. The destination then decides if it should transmit CTS by examining the data and control channels. A source that does not receive CTS in time will back off using a binary exponential algorithm. Once the source receives a CTS message it transmits the data message over the data channel. The destination starts transmitting a busy tone over the control channel once it starts receiving the data message. The main drawback of PAMAS is the inclusion of multiple radios which will greatly increases the energy consumption and the device cost of the sensor network. Additionally, controlling access to two wireless mediums increases the MAC protocol complexity.

Another protocol Is Alert MAC protocol for collecting event-triggered urgent messages from a group of sensor nodes with minimum latency and without requiring any cooperation or pre-scheduling among the senders or between senders and receivers during protocol execution. Alert minimizes contention among nodes by using a combination of time and frequency multiplexing. Multiple frequency channels are used within time slots and contention is minimized by controlling the selection probability of each channel by the nodes. The Alert protocol divides the time into slots which are called Alert slots. Each alert slot can be used to exchange one data packet and its acknowledgment between a sender-receiver pair. In each alert slot, multiple frequency channels can be used by the senders and receivers.

\section{CONCLUSION}

In this paper we have reviewed the main approaches to energy conservation in wireless sensor networks. A systematic and comprehensive classification of the solutions is presented in this paper. The considered approaches are not the alternatives they should rather be exploited together. An important approach that can be considered for energy saving is integration of the outcome of traditional approaches for energy saving. This involves characterizing the interactions between different protocols and exploiting cross-layer interactions. Most of the solutions are based on the assumption that energy consumption of radio is much higher than the consumption in data sampling or processing, while many real applications shows greater power consumption In data sampling/processing instead of radio transmission. Further it is observed that the field of data acquisition has not been fully explored for energy conservation. Finally, I observe an increasing interest towards MAC protocols used for time synchronization and energy conservation. The mobility of nodes is also a challenging task in energy optimization. In many practical applications Microsensor network can be very efficient and robust if communication MAC protocols can appropriately exploit the mobility of collector nodes.

\section{ACKNOWLEDGEMENT}

This work is an outcome of rigorous work done under the supervision and guidance of my research guides. I like to pay my sincere thanks to them, for their kind support and cooperation.

\section{REFERENCES}

[1] A. Deshpande, S. Madden, and MauveDB: supporting model-based user views in database systems, in: Proc. 25th ACM International Conference on Management of Data (SIGMOD'06), Chicago (IL), USA, June 27-29, 2006, pp. 73-84.

[2] A. Ephremides, O. Mowafi, Analysis of a hybrid access scheme for buffered user probabilistic time division, IEEE Transactions on Software Engineering 8 (1) (1982) $52-61$.

[3] A. Keshavarzian, H. Lee, L. Venkatraman, Wakeup scheduling in wireless sensor networks, in: Proc. ACM Mobi Hoc 2006, Florence Italy, May 2006, pp. 322-333.

[4] B. Hohlt, E. Brewer, Network power scheduling for tiny OS applications", in: Proc. 2nd IEEE/ACM International Conference on Distributed Computing in Sensor Systems (DCOSS'06), San Francisco, California, June 2006.

[5] Bashir Yahya and Jalel Ben-Othman , Towards a classification of energy aware MAC protocols for wireless sensor networks WIRELESS COMMUNICATIONS AND MOBILE COMPUTING Wirel. Commun. Mob. Comput. 2009; 9:1572-1607 Published online 4 February 2009 in Wiley InterScience

[6] C. Guo, L. Charlie, Low power distributed MAC for ad hoc sensor radio networks, in: Proc. IEEE Globecom, 2001.

[7] C. Schurgers, V. Tsiatsis, M.B. Srivastava, STEM: topology management for energy efficient sensor networks, in: IEEE Aerospace Conference'02, Big Sky, MT, March 10-15, 2002.

[8] C. Schurgers, V. Tsiatsis, S. Ganeriwal, M.B. Srivastava, Optimizing sensor networks in the energy-latency-density design space, IEEE Transactions on Mobile Computing 1 (1) (2002) 70-8s0.

[9] D. Ganesan, A. Cerpa, W. Ye, Y. Yu, J. Zhao, D. Estrin, Networking issues in wireless sensor networks, Journal of Parallel and Distributed Computing 64 (2004) 799-814.

[10] D.R. Stinson, Combinatorial Designs: Construction and Analysis, Springer, 2003

[11] G. Anastasi, E. Borgia, M. Conti, E. Gregori, A. Passarella, Understanding the real behavior of 802.11 and mote ad hoc networks, Pervasive and Mobile Computing 1 (2) (2005)

[12] G. Anastasi, M. Conti, M. Di Francesco, A. Passarella, How to prolong the lifetime of wireless sensor networks", in: M. Denko, L. Yang (Eds.), Mobile Ad hoc and Pervasive Communications, American Scientific Publishers, in press (Chapter 6). http://info.iet.unipi.it/ $\sim$ anastasi /papers /Yang.pdf.

[13] G. Anastasi, M. Conti, M. Di Francesco, A. Passarella, An adaptive and low-latency power management protocol for 
wireless sensor networks, in: Proc 4th ACM International Workshop on Mobility Management and Wireless Access (MobiWac 2006), Torremolinos, Spain, October 2, 2006.

[14] I. Demirkol, C. Ersoy, and F. Alagoz, MAC Protocols for Wireless Sensor Networks: A Survey, IEEE Communications Magazine, April 2006

[15] IEEE Pervasive Computing, Energy Harvesting and Conservation, vol. 4, issue 1, January-March 2005.

[16] I.F. Akyildiz, W. Su, Y. Sankarasubramaniam, E. Cayirci, Wireless sensor networks: a survey, Computer Networks 38 (4) (2002).

[17] I. Rhee, A. Warrier, M Aia, J. Min, Z-MAC: a hybrid MAC for wireless sensor networks, in: Proc. ACM SenSys 2005, S Diego, USA, November 2005.

[18] J. Rabaey, J. Ammer, T. Karalar, S. Li, B. Otis, M. Sheets, T. Tuan, Picoradios for wireless sensor networks: the next challenge in $566 \mathrm{G}$. Anastasi et al. / Ad Hoc Networks 7 (2009) 537-568 ultra-low-power design, in: Proc. International Solid-State Circuits Conference, San Francisco, CA, February 3-7, 2002.

[19] J. R. Jiang, Y. C. Tsang, C.S. Hsu, T. H. Lai, QuorumBased Asynchronous Power-Saving Protocols for IEEE 802.11 Ad Hoc, Proceedings of the 2003 International Conference on Parallel Processing (ICPP'03) 2003 IEEE.

[20] L. Gu, J. Stankovic, Radio-triggered wake-up for wireless sensor networks, Real-Time Systems Journal 29 (2005) $157-182$.

[21] L. Wang, Kai Liu, An adaptive energy efficient and lowlatency Mac protocol for wireless sensor networks, IEEE 2007.

[22] L. Zhang and Z. Wang, Integration of RFID into Wireless Sensor Networks: Architectures, Opportunities and Challenging Problems Proceedings of the Fifth International Conference on Grid and Cooperative Computing Workshops (GCCW'06) IEEE 2006

[23] Mário Macedo, António Grilo, Mário Nunes, Distributed Latency-Energy Minimization and interference avoidance in TDMA Wireless Sensor Networks, Elsevier Journal on Computer Networks 53 (2009) 569-582

[24] P. Buonadonna, D. Gay, J. Hellerstein, W. Hong, S. Madden, TASK: sensor network in a box, in: Proc. European Workshop on Sensor Networks (EWSN 2005), January 2005.

[25] P. P. Czapski, A Survey: MAC Protocols For Applications Of Wireless Sensor Networks, IEEE 2006.

[26] R. Willett, A. Martin, R. Nowak, Backcasting: adaptive sampling for sensor networks, in: Proc. Third International Symposium on Information Processing in Sensor Networks (IPSN 2004), 26-27 April, 2004, pp. 124-133.

[27] R. Zheng, J. Hou, L. Sha, Asynchronous wakeup for ad hoc networks, in: Proc. ACM Mobi Hoc 2003, Annapolis, USA, June 1-3, 2003, pp. 35-45.
[28] S. C. Ergen, P. Varaiya, TDMA scheduling algorithms for wireless sensor networks, Springer Wireless Network (2010) 16:985-997.

[29] S. Goel, A. Passarella, T. Imielinski, Using buddies to live longer in a boring world, in: Proc. IEEE International Workshop on Sensor Networks and Systems for Pervasive Computing (PerSeNS 2006), Pisa, Italy, March 13, 2006.

[30] S. Madden, M. Franklin, J. Hellerstein, W. Hong, TAG: a tiny aggregation service for ad hoc sensor networks, in: Proc. Annual Symposium on Operating Systems Design and Implementation (OSDI), 2002.

[31] S. Madden, M.J. Franklin, J.M. Hellerstein, W. Hong, TinyDB: an acquisitional query processing system for sensor networks, ACM Transactions on Database Systems 30 (1) (2005) 122-173.

[32] Singh S, Raghavendra C. PAMAS: power aware multiaccess protocol with signaling for ad hoc networks. SIGCOMM Computer Communications Review 1998; 28(3): 5-26

[33] T.Armstrong, Wake-up based power management in multi-hop wireless networks. http://www.eecg.toronto. edutrevor/Wakeup/index.html.

[34] T.V. Dam and K. Langendoen, An adaptive energyefficient MAC protocol for wireless sensor networks, in: The First ACM Conference on Embedded Networked Sensor Systems (Sensys'03), Los Angeles, CA, USA, November 2003.

[35] V. Paruchuri, S. Basavaraju, R. Kannan, S. Iyengar, Random asynchronous wakeup protocol for sensor networks, in: Proc. IEEE International Conference on Broadband Networks (BROADNETS 2004), 2004.

[36] V. Raghunathan, C. Schurghers, S. Park, M. Srivastava, Energy-aware wireless micro sensor networks, IEEE Signal Processing Magazine (2002) 40-50.

[37] W. L. Lee, A Datta FlexiTP: A Flexible-Schedule-Based TDMA Protocol for Fault-Tolerant and Energy-Efficient Wireless Sensor Networks IEEE Transactions on Parallel and Distributed System, vol 19, No 6, June 2008.

[38] W. Ye, J. Heidemann, D. Estrin, Medium access control with coordinated adaptive sleeping for wireless sensor networks, IEEE/ACM Transactions on Networking 12 (3) (2004) 493-506.

[39] X. Yang, N. Vaidya, A wakeup scheme for sensor networks: achieving balance between energy saving and end-to-end delay, in: Proc. IEEE Real-Time and Embedded Technology and Applications Symposium (RTAS 2004), 2004, pp. 19-26. 